\title{
Neues Konzept ermöglicht weniger Inhaltsstoffe
}

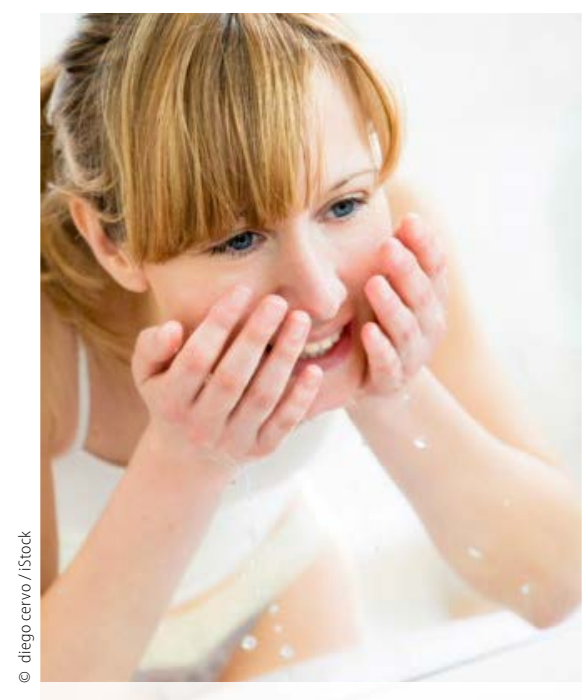

Die sterile Kosmetik bietet eine sichere und nebenwirkungsarme Anwendung.
Pierre Fabre, seit mehr als 50 Jahren Pionier auf dem Gebiet der Dermo-Kosmetik, entwickelte das neue Konzept der sterilen Kosmetik. Ein Sterilisierungsverfahren gepaart mit einem patentierten Tubenverschlusssystem ermöglicht Produkte ohne Konservierungsstoffe. Damit erlaubt das neue Verfahren die Konzentration auf essenzielle Inhaltsstoffe für die Haut. Höchste Sicherheit und Hautverträglichkeit der Produkte sind das Ergebnis.

Vorrangiges Kriterium bei Hautpflegeprodukten ist die sichere und nebenwirkungsfreie Anwendung. Zum Schutz der Formulierung vor Kontamination und Oxidation werden häufig Konservierungsstoffe zugeführt, die das Keimwachstum hemmen und die Haltbarkeitsdauer verlängern sollen. Nebenwir- kungen wie Kontaktekzeme, Allergien und Überempfindlichkeitsreaktionen können auftreten, sobald die Konservierungsstoffe von der Haut aufgenommen werden. Die Technologie von Pierre Fabre Dermo-Kosmetik garantiert die Herstellung und Abfüllung der Produkte unter sterilen Bedingungen. Auf Konservierungsstoffe kann dadurch verzichtet werden. Das patentierte und luftdichte D.E.F.I(Device for Exclusive Formula Integrity)-Dosiersystem schützt die Pflegeprodukte vor dem Eindringen von Bakterien. Der speziell entwickelte Tubenverschluss gewährleistet, dass die Formulierungen steril abgefüllt werden und die Produkte während der gesamten Anwendungsdauer keimfrei bleiben.

Sterile Kosmetikprodukte werden bereits in den Produktlinien Avène und A-Derma angeboten. Seit März 2016 ist die Eau Thermale Avène Pflegelinie Tolérance Extrême neu formuliert und um zwei Pflegeprodukte ergänzt in Apotheken erhältlich.

Nach Informationen von Pierre Fabre

\section{Neue Ansätze zur Rejuvenation und Gesichtskonturierung}

Kollagenverlust und subdermale Prozesse wie Fettabbau und das Absacken von Fettdepots sind die Hauptfaktoren des Alterungsprozesses im Gesicht. Mit seinen Produkten Silhouette Soft ${ }^{\oplus}$, Ellansé ${ }^{m}$ und Sculptra ${ }^{\circledast}$ berücksichtigt Sinclair Pharma die natürlichen hauteigenen Prozesse, um den Zustand der Haut zu erhalten und zu verbessern und für eine verjüngte Ausstrahlung des Gesichts zu sorgen.

„Während sich das Fadenlifting einer immer größeren Beliebtheit erfreut, ist das Konzept der Kollagenstimulation für viele Ärzte noch immer Neuland. Rund $90 \%$ der angebotenen VolumisierungsPräparate sind dem Bereich der reinen Hyaluronsäurefiller zuzuordnen. Dabei

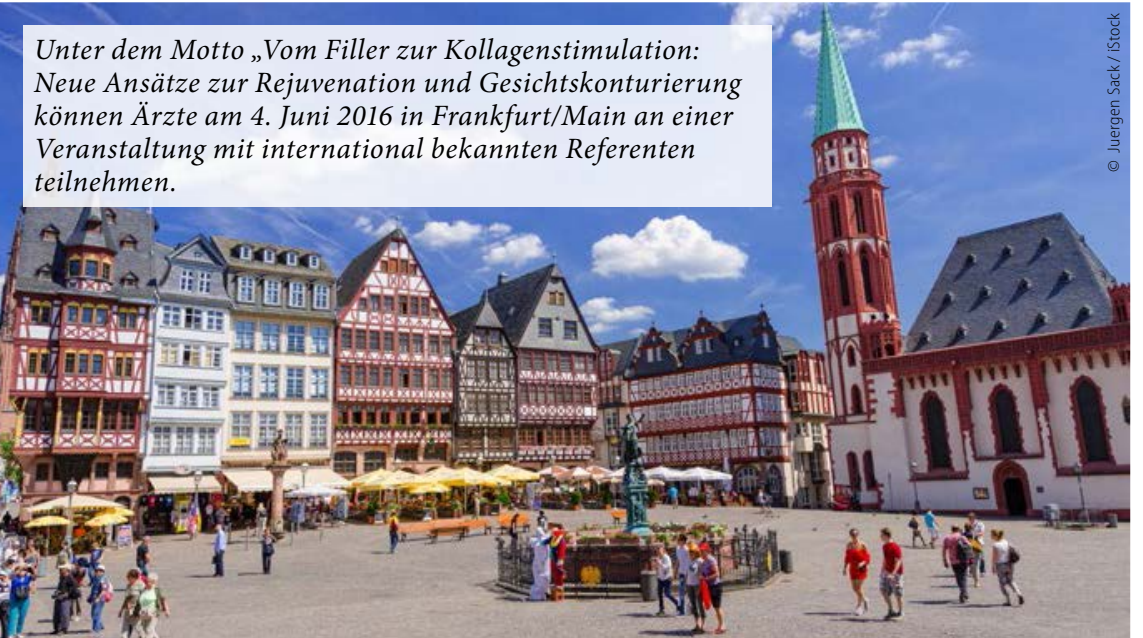

liegen die Vorteile auf der Hand: Viele Patienten schätzen die längere Wirksamkeit der Kollagenstimulatoren“, sagt Alexandra Schubert, Geschäftsführerin von Sinclair Pharma Deutschland.

Wer mehr über das Thema erfahren möchte, kann dies am 4. Juni 2016 in der Villa Kennedy in Frankfurt/Main tun. Unter dem Motto „Vom Filler zur Kollagenstimulation und Fadenlifting: Neue Ansätze zur Rejuvenation und Gesichtskonturierung" lädt das Unternehmen zum ersten Sinclair Expert Day ein. Der hauseigene Kongress wartet mit international bekannten Experten wie Dr. Roberto Pizzamiglio (Marbella), Dr. Inigo de Felipe (Barcelona), Prof. Dr. Alain Mojallal (Lyon) und Dr. Danny Vleggar (Genf) auf. Neben wissenschaftlich-kurzweiligen Vorträgen erwarten die Teilnehmer spannende Live-Demos in englischer Sprache. Da ein reger Austausch mit den Dozenten ausdrücklich erwünscht ist, wird simultan übersetzt.

Weitere Informationen zum ersten Sinclair Expert Day erhalten Sie unter www.sinclairpharma.de/sinclair-expertday oder Telefon 069/710 456330.

Nach Informationen von Sinclair Pharma 